\title{
Optical and Infrared Photometric Study of Old Open Star Cluster Berkeley 39
}

\author{
A. Essam1, I. M. Selim 1,2 \\ ${ }^{1}$ Astronomy Department National Research Institute of Astronomy and Geophysics (NRIAG), Cairo, Egypt \\ ${ }^{2}$ Computer Science Department Higher Technological Institute (HTI), Cairo, Egypt \\ Email: i selim@yahoo.com
}

Received 5 June 2015; accepted 25 September 2015; published 28 September 2015

Copyright (C) 2015 by authors and Scientific Research Publishing Inc.

This work is licensed under the Creative Commons Attribution International License (CC BY).

http://creativecommons.org/licenses/by/4.0/

(c) (i) Open Access

\begin{abstract}
In this paper we present astrophysical parameters of the very old open star cluster Berkeley 39 using 2MASS JHK data and observation of BV CCD photometry ( $\sim 21.5 \mathrm{mag}$ ) in Johnson-Cousin filters. We have derived reddening, distance, metallicity and age etc. using two color diagrams in near-infrared and optical bands. We have found $E_{\mathrm{B}-\mathrm{v}}=0.099 \pm 0.02, E_{\mathrm{J}-\mathrm{H}}=0.018 \pm 0.001$ and $E_{\mathrm{J}-\mathrm{Ks}}=$ $0.016 \pm 0.001 \mathrm{mag}$, and the distance modulus of $(\mathrm{m}-\mathrm{M})_{0}=13.24 \pm 0.03 \mathrm{mag}$, which give a distance of $4.2 \pm 0.04 \mathrm{Kpc}$, and the age of the cluster is $5.6 \mathrm{Gyr}$ with metallicity; $Z=0.008$ (obtained from fitting of the observed CMDs with Padova isochrones). The radius of the cluster obtained from the analysis of 2 MASS data is equal $9.0 \pm 0.13$ arc minutes; it reflects that the cluster have large radius with new cluster center. Luminosity and mass functions have been investigated as well as the dynamical relaxation time of the cluster Berkeley 39.
\end{abstract}

\section{Keywords}

Galaxy, Open Clusters, Associations, Individual, Berkeley 39

\section{Introduction}

Open star clusters are the excellent tool to trace the properties of star formation, the formation and the evolution of the disc of our galaxies and structural points of view. Studying the old open star cluster trace back the first moments of the disc lifetime and putting crucial constraints on its formation mechanism and early evolution of the open cluster system, all this allow us to explore the history of star and cluster formation over wide range of the galaxy.

Old open star cluster allow us to investigate the chemical evaluation of the galaxies and stage of formation the galactic disk. Many detailed studies of the old open clusters [1]-[6]. The oldest open star cluster Berkeley 39 is 
located in the outer part of the disc, about $11 \mathrm{kpc}$ from the galactic center, [5] and reference there in, at $(\alpha 2000.0=$ $07 \mathrm{~h} 47 \mathrm{~m} 18 \mathrm{~s}, \delta 2000.0=-04^{\circ} 44^{\prime}, \mathrm{l}=223^{\circ}, \mathrm{b}=10^{\circ}$ ). [1] find that the total absorption in the direction of the cluster Berkeley $39 E_{(\mathrm{B}-\mathrm{V})}=0.15 \pm 0.02$ and dated that the cluster age about 8 Gyr with $E_{\mathrm{B}-\mathrm{v}}=0.12$ and $(\mathrm{m}-\mathrm{M})_{\mathrm{v}}$ $=13.4 \mathrm{mag}$, based on BV CCD observation. The mean velocity of Berkeley 39 is $+55 \pm 10$ as determined by [7]. [3], determined the datasets for Berkeley 39 are: age is $6 \pm 2 \mathrm{Gyr}$ and $(\mathrm{m}-\mathrm{M})_{0}=12.9 \pm 0.2$ mag with adapted the reddening $\mathrm{E}_{\mathrm{B}-\mathrm{V}}=0.11 \pm 0.09$.

The database of cluster Berkeley 39 published in the WEBDA is Reddening $=0.12$ mag., log Age $=9.9$ Gyr, with Distance modulus $=13.8 \mathrm{mag}$, Metallicity $=-0.17$ and Distance $=4.7 \mathrm{kPc}$. [8] find the reddening of $\mathrm{E}_{(\mathrm{B}-\mathrm{V})}=$ $0.11 \pm 0.09$ using $\mathrm{H} \beta$ line for blue stars in the cluster field. Also [9] determines the chemical composition of Berkeley 39 from high-resolution spectra of giants and finds that the cluster is slightly metal-poor $([\mathrm{Fe} / \mathrm{H}]=$ $-0.21 \pm 0.01$ ). 2MASS JHK bands beside CCD photometry ( $\mathrm{V} \sim 21.5 \mathrm{mag}$ ) is taken in BV Johnson-Cousin filters which enable us to properly investigate photometric and structure parameters of the clusters.

Old open star cluster is very important objects, however, Berkeley 39 is important to re-investigate its position and age, which are estimated in a precision way from their Color-Magnitude Diagrams using the most recent Near-Infrared photometric data from the 2-Micron All Sky Survey (2MASS). Other motivation of this project is to study the useful parameters to improve our knowledge about the distance scale and the kinematic properties and structures of the Milky Way Galaxy.

The paper is organized as follows: the observations and data reduction are described in Section 2. The analysis of the color-magnitude diagrams and the derived photometric parameters are given in Section 3. The Cluster Membership is presented in Section 4. Radial density distribution is presented in Section 5. The luminosity function and mass function are described in Section 6, and mass segregation and dynamical relaxation time are described in Section 7. The conclusion and summary are presented in last section.

\section{Observations and Data Reduction}

The cluster was observed with the direct camera of the $188 \mathrm{~cm}$ reflector telescope at Newtonian focus (f/4.9) during one night on 2013, March 19, in BV CCD images. The telescope is operating by Kottamia observatory, astronomy department of NRIAG, Egypt. It was equipped with a $2 \mathrm{k} \times 2 \mathrm{k}$ EEV $42-40$ CCD camera, its pixel size of $13.5 \mu \mathrm{m}$. The telescope and this CCD camera give us a square field of view of about $10 \times 10 \operatorname{arcmin}^{2}$ on the sky with a scale of 0.30 arc sec per pixel, more information about the capabilities of Kottamia telescope are presented in [10]. Log of observation gives in Table 1, the numbers of observations for each filter, exposer times and seeing are listed. Bias and twilight flat field frames were taken in the same period and same filters of observation. The observations of the standard stars in the selected area SA 107, [11] have been performed to calibrate the target observations also in the same period of observations [12].

All CCD reductions for the cluster and standard stars were done using IRAF. These processes include overscan, bias subtraction, flat field corrections, and removal of cosmic rays in the standard way. 20 sky flat field were used to flat field the object and standard fields. The instrumental magnitude processes of the cluster were obtained using point spread function using IRAF photometry tasks. The magnitude transformations to the standard system (Londolt 1992) also have been done using IRAF tasks [12]. The transformation equations are in the following form:

$$
\begin{aligned}
& b=B+z_{b}+(k \times X)+a_{b} \times(\mathrm{B}-\mathrm{V}) \\
& v=V+z_{v}+\left(k_{v} \times X\right)+a_{v} \times(\mathrm{B}-\mathrm{V})
\end{aligned}
$$

where $B, V$, and $b, v$, are the standard and the instrumental magnitudes respectively. $X$ is the airmass, while $z_{b}, z_{v}$, and $k_{b}, k_{v}$, are the photometric zero points and the extinction coefficients in $B, V$ filters respectively. The $a_{b}, a_{v}$, are the color coefficients. The values of extinction coefficients, the color coefficients and the photometric zero points in their corresponding filters are given in Table 2. The near-infrared JHKs filters (2MASS) photometric data were extracted from Vizier web page for the star cluster Berkeley 39.

\section{The Color Magnitude Diagrams}

The most important parameter in the cluster is the age and distance. Color-Magnitude-Diagram (CMD) has been used to determine the age, reddening and distance. So, the optical $(\mathrm{V}, \mathrm{B}-\mathrm{V})$, and infrared $(\mathrm{J}, \mathrm{J}-\mathrm{H}),(\mathrm{H}, \mathrm{J}-\mathrm{H})$, and 
Table 1. Log of observations.

\begin{tabular}{ccccc}
\hline Date & Filter & No. of exp. & Air mass range & Exp. T sec \\
\hline March 19, 2013 & $B$ & 12 & $1.215-1.506$ & 300 \\
March 19, 2013 & $V$ & 2 & $1.204-1.258$ & 180 \\
March 19, 2013 & $V$ & 4 & $1.206-1.245$ & 300 \\
March 19, 2013 & $V$ & 6 & $1.258-1.402$ & 360 \\
\hline
\end{tabular}

Table 2. The color and extinction coefficients and zero point, for each filter.

\begin{tabular}{cccc}
\hline Filter vs. parameter & $K$ & $\mathrm{a}$ & $\mathrm{z}$ \\
\hline$B$ & 0.38 & 0.023 & 3.24 \\
$V$ & 0.21 & 0.031 & 3.1 \\
\hline
\end{tabular}

$\left(K_{s}, \mathrm{~J}-\mathrm{H}\right)$ CMDs for the total number of the observed stars in the decontaminated cluster region $(r<$ rlim) are constructed for the cluster Berkeley 39 and presented in Figure 1 \& Figure 2. The CMDs were fitted with several theoretical Padova isochrones [13], to obtund fundamental parameters of the cluster, these in different metallicities and ages in steps of 0.05 in the logarithm of age. This step was adopted as a typical uncertainty of the $\log$ age. We got a good fit for all CMDs by visual inspection with the isochrones of the metallicity $Z=0.008$, and the corresponding age of 5.6 Gyr. The optical and infrared color excesses are determined from CMDs fitting, the optical color excess $E_{\mathrm{B}-\mathrm{V}}=0.099 \pm 0.02$, and the color excess values for 2MASS $E_{\mathrm{J}-\mathrm{H}}=0.018 \pm 0.001$ and $E_{\mathrm{J}-\mathrm{Ks}}=0.016 \pm 0.001 \mathrm{mag}$, the distance modulus of $(\mathrm{m}-\mathrm{M})_{0}=13.24 \pm 0.03 \mathrm{mag}$, which gives a distance of $4.2 \pm$ $0.04 \mathrm{Kpc}$.

Some of these results agree well with which given by [1], dated that the total absorption in the direction of the cluster Berkeley $39 E(\mathrm{~B}-\mathrm{V})=0.15 \pm 0.02$ and dated that the cluster age about 8 Gyr with $E_{\mathrm{B}-\mathrm{V}}=0.12$ and $(\mathrm{m}-\mathrm{M}) \mathrm{v}=13.4$ mag., While [2], determined the datasets for Berkeley 39 are: age is $6 \pm 2 \mathrm{Gyr}$ and $(\mathrm{m}-\mathrm{M}) \mathrm{o}=$ $12.9 \pm 0.2 \mathrm{mag}$, The reddening $E_{\mathrm{B}-\mathrm{V}}=0.11 \pm 0.09$ determined by [2] [7] found the reddening of $E(\mathrm{~B}-\mathrm{V})=0.11 \pm$ 0.09 using $\mathrm{H} \beta$ line for blue stars in the cluster field.

\section{Photometric Selection of Candidate Cluster Members}

Determination membership of stars cluster from field stars is an essential task to obtain an accurate photometric study for star clusters. To decontaminate the membership stars in a cluster, we taking in account the area of the optical field of view is small comparing to 2MASS area, we used a technique given by [14] as described in the following steps:

a) In the $V$ vs. B-V color magnitude diagram in Figure 1, the stars have been selected as probable members all those stars which according to their $\sigma V$ and $\sigma \mathrm{B}-\mathrm{V}$ errors, belong to a well-defined strip in the CMD. The envelope of this strip follows the representative main sequence for the cluster.

b) We considered at first the stars chosen above and have 2MASS photometry available along an analogous strip, in the $J$ vs. J-H color-magnitude diagram, and rejected from our sample of initial possible candidate members stars which, have distance from the center less than cluster region $(r<\operatorname{rlim})$ are constructed cluster member.

c) Finally, we considered as possible cluster candidate members those belonging to the strip defined in the $V$ vs. B-V and $J$ vs. J-H colors magnitude diagram. The star member in the 2MASS data more than the optical one due to the difference in field of view.

\section{Cluster Center \& Radial Density Distribution}

Due to the small area of the optical field of view, we investigate the cluster structure based only on the 2MASS data, starting with determination of the cluster center. The cluster center determination by constructing a rectangular strip around the cluster center (visually determined) in $\alpha$ and $\delta$ direction with a side of one degree, we count the stars in each strip to build the frequency distribution in both directions. The histograms were fitted by a Gaussian function, whereas the location of maximum number of stars (peak) indicates the new cluster center, 


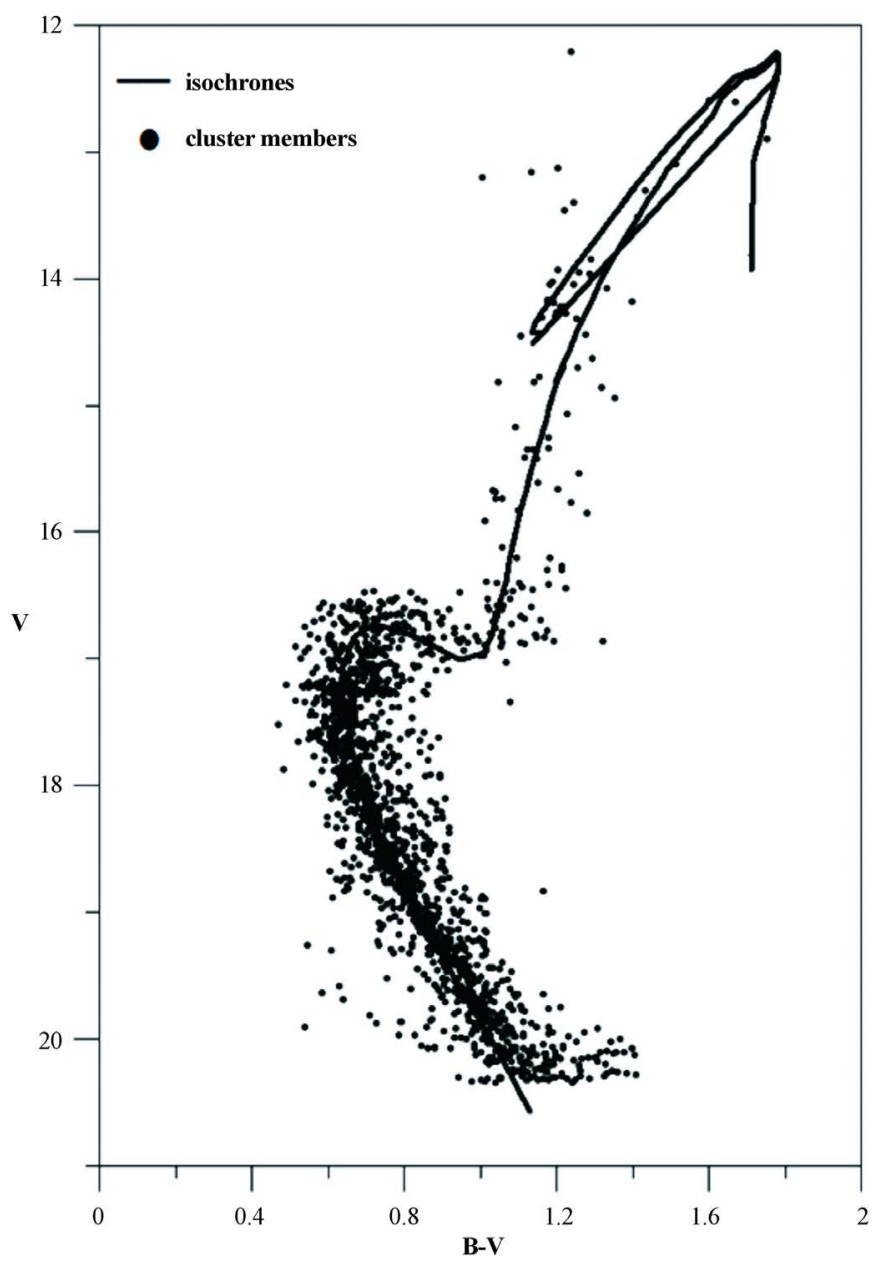

Figure 1. Color magnitude diagrams $(V, \mathrm{~B}-\mathrm{V})$, the solid curve represents the best fitted Padova isochrones with $z=0.008$ and age of $5.6 \mathrm{Gyr}$.

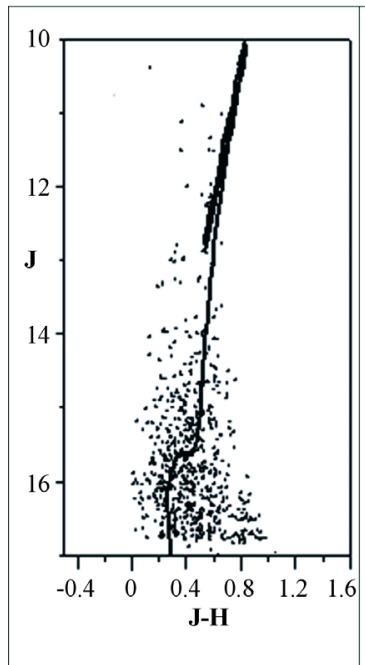

(a)

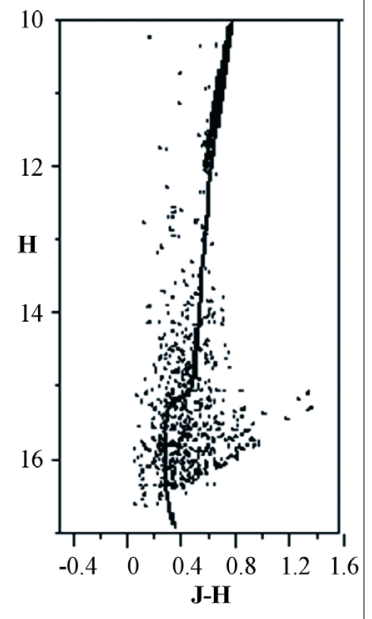

(b)

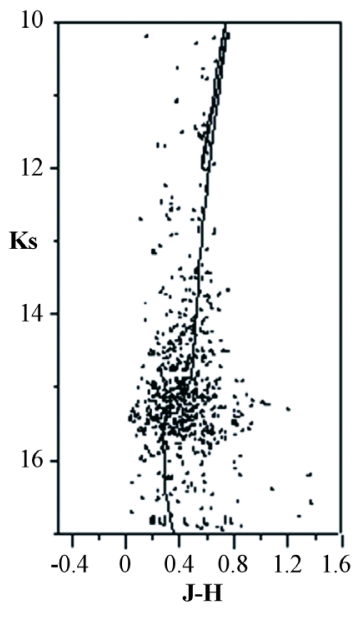

(c)

Figure 2. 2MASS CMDs. (a) $(J, \mathrm{~J}-\mathrm{H})$; (b) $(H, \mathrm{~J}-\mathrm{H})$; and $(\mathrm{c})\left(K_{s}, \mathrm{~J}-\mathrm{H}\right)$, the solid curve represents the best fitted Padova isochrones with $z=0.008$ and age of $5.6 \mathrm{Gyr}$, and black points are cluster members. 
at $\left(\alpha 2000.0=07 \mathrm{~h} 46 \mathrm{~m} 42 \mathrm{~s}, \delta 2000.0=-04^{\circ} 39^{\prime}, \mathrm{l}=223.29^{\circ}, \mathrm{b}=10.02^{\circ}\right)$, as shown in Figure 3.

The internal and external dynamical process is affected of stellar density distribution of a star in the cluster. A good way to investigate the evidence of extra tidal extension in the cluster is the radial surface density profile of the cluster (the spatial distribution of stars around the cluster center) [15]. We examined the radial surface density distribution of stars as a function of distance from the center of the cluster. The number of stars per unit area at different radius from cluster's center outward is the Radial Density Profile (RDP). The radial density profile is determined through counting the stars in concentric rings around the cluster's center. We calculated the density of each ring by dividing the number of stars in the ring by its area. The ring radius was chosen to be ranged from 0.25 to 15 arc-minutes. We applied the empirical King model [16] to fit the observed RDP. The equation of King model for the density function is in the following form:

$$
\rho(r)=f_{b g}+\frac{f_{0}}{1+\left(\frac{r}{r_{\text {core }}}\right)^{2}}
$$

where $r_{c o r e}$, $f_{0}$, and $f_{b g}$ are the core radius, the central surface density and background surface density and their values of $r_{\text {core }}=3 \pm 0.09$ arcmin, $f_{0}=0.32 .7 \pm 0.20 \mathrm{stars} / \mathrm{arcmin}^{2}$ and $f_{b g}=4.1 \pm 0.21$ stars/arcmin ${ }^{2}$ respectively.

The radius at which the central surface density drops to its half value is called the core radius. The density profile must represent the well exponential decreasing of the cluster surface density outward from its center, as well as the constancy of the dense background after the cluster limit as it has been shown on Figure 4. We suppose that the background star density lies within the lines represent the standard deviation of the background density level, therefore we define the cluster limits (cluster limited radius; $r_{\lim }=9.2 \pm 0.17 \mathrm{arcmin}$ ) at the intersection of the King profile with the upper limit of the density standard deviation line, see Figure 4, the background star density at this point $\left(\rho_{b}=f_{b g}+3 \sigma_{b g}\right.$ ), where $\sigma_{b g}$ is uncertainty of $f_{b g}$.

\section{The Luminosity and Mass Function}

The 2MASS uniform sky coverage has been shown to be an ideal tool for studies the cluster area. In this section we analyze the observed stars counts as a function of magnitude to obtain the Luminosity Function (LF) and Mass Function (MF), as well as their spatial dependence. Near-infrared surveys are very useful for the investigation of clusters. It is relatively less affected by high reddening from the Galactic plane. However, due to the small area of the optical field of view, we investigate the Luminosity Function (LF) based on the 2MASS data, it

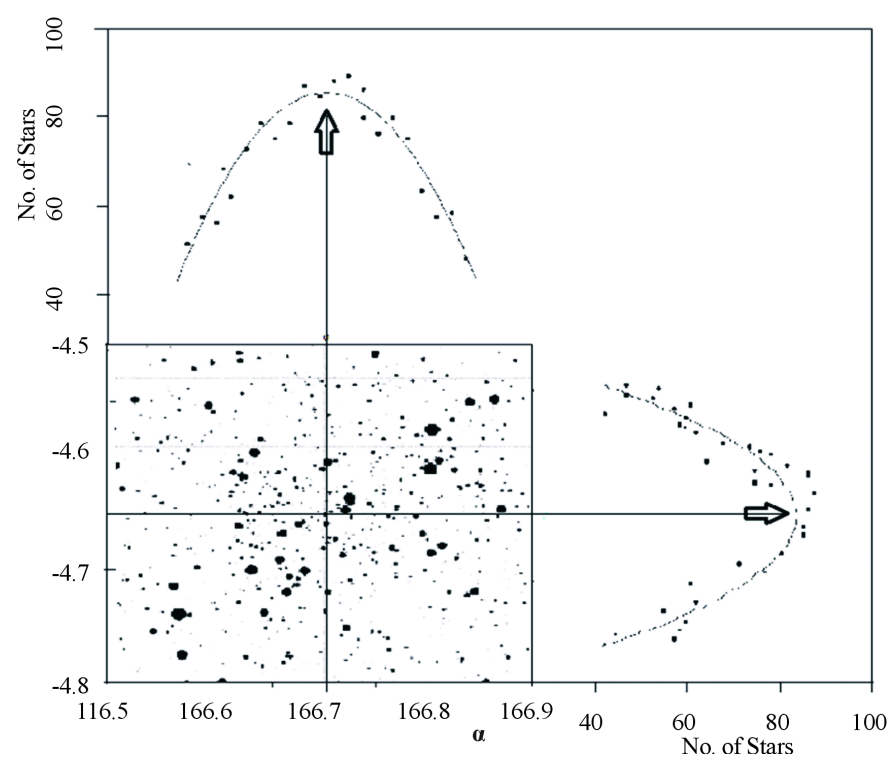

Figure 3. The Gaussian fitting profiles of star counts across Right Ascension $(\alpha)$ and Declination $(\delta)$ for the cluster. The peak's position of the profile gives the cluster's center. 


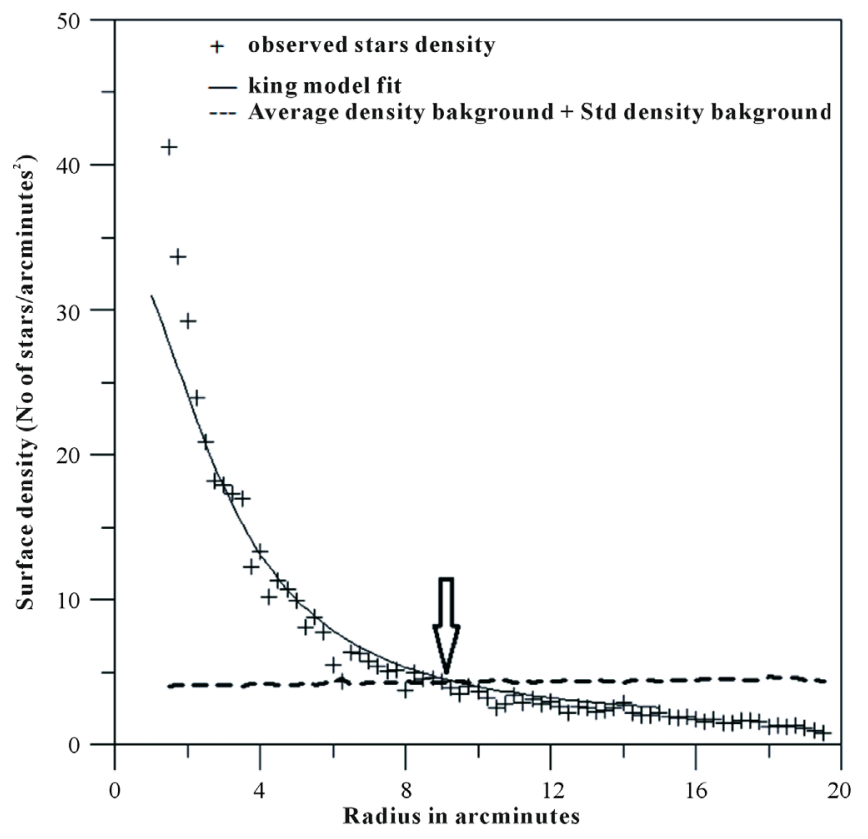

Figure 4. Radial density profile of the cluster, the solid curve is the fitting with King Model, while the dashed lines mark the level of the background density + standard deviation of the background density.

is given in terms of the absolute magnitude (MJ), obtained after applying the distance modulus derived in Section 3, the frequency distribution of the $J$ absolute magnitude has been obtained, as shown in Figure 5, and the bin used is $\Delta \mathrm{MJ}=1 \mathrm{mag}$. LFs inside Berkeley 39 have been built following the structures present in the radial density profile (Figure 4).

The mass function (MF) is obtunding from (LF) using the theoretical evolutionary tracks and their isochrones with different ages selected [13]. Masses of possible cluster members were derived from the polynomial expression developed by [17] with solar metallicity. The absolute magnitude (MJ) and the masses for the adopted isochrones with metallicity $Z=0.008$ are used to construct the relation between $\mathrm{M}_{\odot}$ and absolute magnitude. The relation is a polynomial function, used to determine masses of the cluster from the observed absolute magnitude (MJ), then the number of stars as a function of mass interval was preformed and presented in Figure 6. It is clear that the MFs shown in Figure 6, being flat in the core of the cluster region and very steep in the outer cluster region $\log \left(\mathrm{M} / \mathrm{M}_{\odot}\right) \geq 1$. The mass frequency distribution is linearly fitted and it is noted that the investigated MF slope value of -4.808 , the properties and evolution of a star are closely related to its mass.

\section{Mass Segregation and Dynamical Relaxation Time}

Any stellar system may be characterized by the dynamical time scales (the two body relaxation time) and mass segregation of star cluster, which is an important tool to investigate the star forming process and dynamical evolution of star clusters. The mass segregation resulting from star formation, fast dynamical evolution of star from their parent molecular cloud, i.e. the distribution of masses of the stars along the radius of the cluster showed a clear segregation of mass in the cluster, with increased concentration of massive stars towards the centre. The relaxation time is the most important vector in the dynamics of any stellar system. After a long enough time, the star's perpendicular speed will (on average) grow to equal its original speed, which is called the relaxation time. We have to estimate the relaxation time to decide whether the mass segregation is primordial or due to dynamical relaxation. To compute the dynamical relaxation time for the clusters, $T_{E}$, we have used the relation given by [18].

$$
T_{E}=\frac{8.9 \times 10^{5} N^{1 / 2} R_{h}^{3 / 2}}{\langle m\rangle^{1 / 2} \log (0.4 N)}
$$




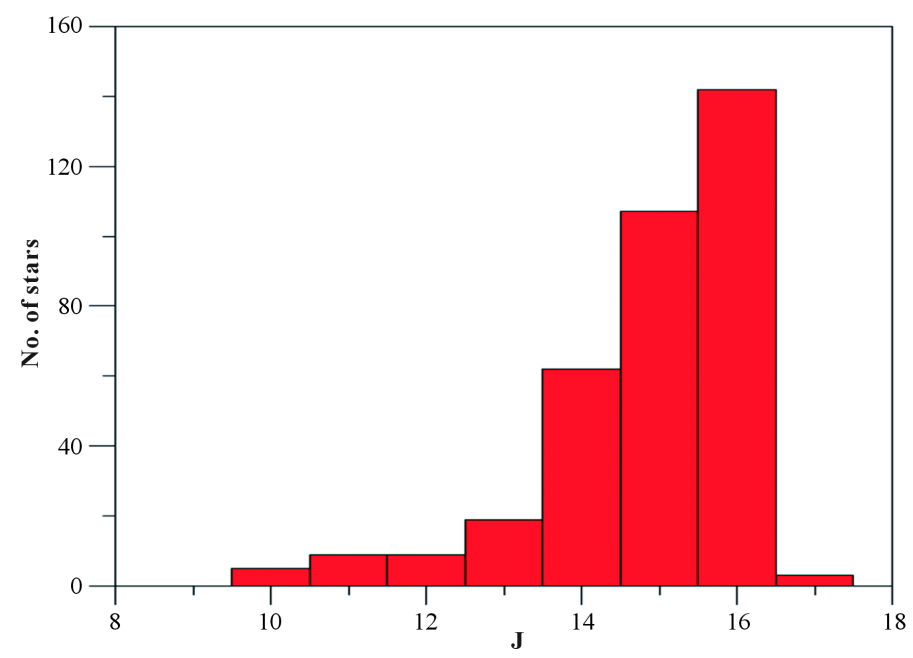

Figure 5. The luminosity function of the cluster.

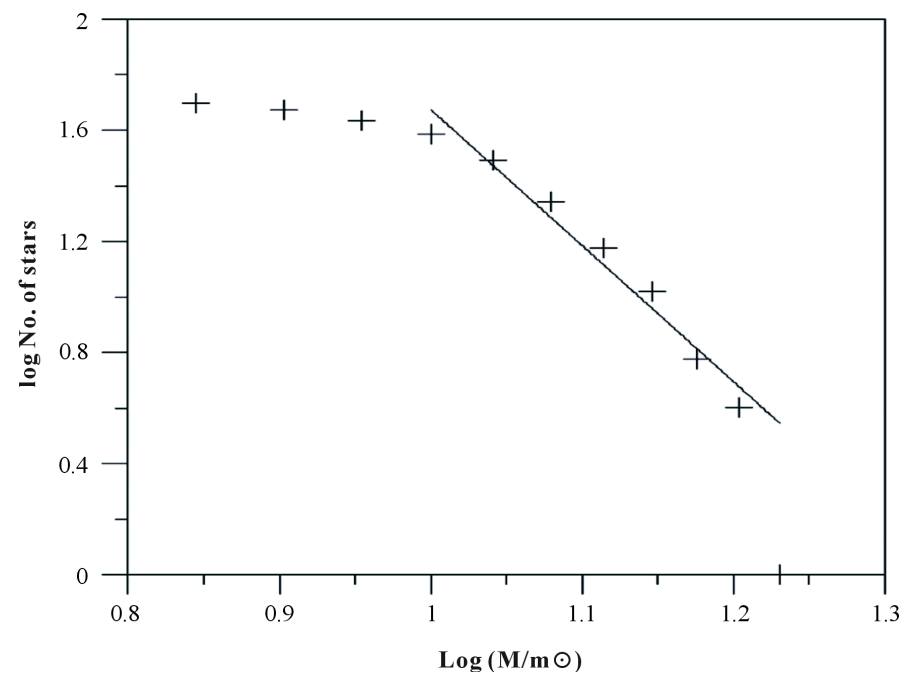

Figure 6. The mass frequency distribution is linearly fitted.

Where $R_{h}$ is the radius containing half the cluster mass, $N$ is the number of cluster members, and $<m>$ is the average star mass in the cluster. The calculated dynamical relaxation time ( $\left.T_{E}=66.22 \mathrm{Myr}\right)$ with $R_{h}$ equal to half of the cluster radius in linear units. We have been comparing the values of $T_{E}$ with the cluster ages, we find Age $/ T_{E}=78.4$ given the fact that the relaxation times are shorter than the cluster age due to the evaluation of the system and the cluster is more relaxed and stable.

\section{Conclusion}

In the present work, the observation of open star clusters Berkeley 39 in optical BV and JHKs 2MASS (infrared) data is used to determine the cluster structure and photometric parameters. The optical and infrared dataset reveals that the cluster is very old open star cluster, and rich star cluster and the cluster in stability and relaxed star cluster. The astrophysical parameters have been estimated some of these parameters spatial infrared parameters for the first time for these clusters. The main parameters are summarized and listed in Table 3.

\section{Acknowledgements}

This paper is a part of the project No. STDF-1335; funded by Science \& Technology Development Fund (STDF) under the Egyptian Ministry for Scientific Research. The project team expresses their deep appreciation to the 
Table 3. The astrophysical parameters of the cluster.

\begin{tabular}{cc}
\hline Parameter & Value \\
& $\mathrm{A}=07 \mathrm{~h} 46 \mathrm{~m} 42 \mathrm{~s}$ \\
& $\delta=-04^{\circ} 39^{\prime}$ \\
$\mathrm{l}=223.29^{\circ}$ & $\mathrm{b}=10.02^{\circ}$ \\
& $9 \pm 0.13$ \\
$R$ lim arc minute & $3 \pm 0.09$ \\
$R$ core arc minute & $0.016 \pm 0.001$ \\
$\left.E_{(\mathrm{J}-\mathrm{ks}}\right)$ mag. & $0.018 \pm 0.001$ \\
$E_{(\mathrm{J}-\mathrm{H})}$ mag. & $0.099 \pm 0.02$ \\
$E_{(\mathrm{B}-\mathrm{V})}$ mag & 4200 \\
Distance (pc.) & 5.6 \\
Age (Gyr.) & $13.24 \pm 0.03$ \\
$(\mathrm{~m}-\mathrm{M})_{0}$ mag. & 66.22 \\
Relax Time (Myr.) &
\end{tabular}

administrators of STDF and its organization. This publication also makes use of data products from the Two Micron All Sky Survey, which is a joint project of the University of Massachusetts and the Infrared Processing and Analysis Center/California Institute of Technology, funded by the National Aeronautics and Space Administration and the National Science Foundation”.

\section{References}

[1] Janusz, K. and Richtler, T. (1989) CCD BV Photometry of the Old Open Cluster Berkeley 39. Acta Astronomica, 39, 139-151.

[2] Kassis, M., Janes, K.A., Friel, E.D. and Phelps, R.L. (1997) Deep CCD Photometry of Old Open Clusters. Astronomical Journal, 113, 1723-1732. http://dx.doi.org/10.1086/118388

[3] MacMinn, D., Phelps, R.L., Janes, K.A. and Friel, E.D. (1994) Berkeley 20: An Unusual Old Open Cluster. Astronomical Journal, 107, 1806-1813. http://dx.doi.org/10.1086/116989

[4] Blake, R.M. and Rucinski, S.M. (2004) Photometry and Spectroscopy of Short-Period Binary Stars in Four Old Open Clusters. Bulletin of the American Astronomical Society, 36, 1483.

[5] Bragaglia, A., Gratton, R.G., Carretta, E., D’Orazi, V., Sneden, C. and Lucatello, S. (2012) Searching for Multiple Stellar Populations in the Massive, Old Open Cluster Berkeley 39.

[6] Carraro, G., de Silva, G., Monaco, L., Milone, A.P. and Mateluna, R. (2014) Updated Properties of the Old Open Cluster Melotte 66: Searching for Multiple Stellar Populations. Astronomy \& Astrophysics.

[7] Friel, E.D. and Janes, K.A. (1993) Metallicities and Radial Velocities of Old Open Clusters. Astronomy \& Astrophysics.

[8] Friel, E.D., Janes, K.A., Tavarez, M., Scott, J., Katsanis, R., Lotz, J., Hong, L. and Miller, N. (2002) Metallicities of Old Open Clusters. Astronomical Journal, 124, 2693. http://dx.doi.org/10.1086/344161

[9] Friel, E.D., Jacobson, H.R. and Pilachowski, C.A. (2010) Abundances of Red Giants in Old Open Clusters. V. Be 31, Be 32, Be 39, M 67, NGC 188, and NGC 1193. Astronomical Journal, 139, 1942. http://dx.doi.org/10.1088/0004-6256/139/5/1942

[10] Azzam, Y.A., Ali, G.B., Ismail, H.A., Haroon, A. and Selim, I. (2010) Current and Future Capabilities of the 74-Inch Telescope of Kottamia Astronomical Observatory in Egypt. Third UN/ESA/NASA (Workshop on the International Heliophysical Year 2007).

[11] Landolt, A.U. (1992) UBVRI Photometric Standard Stars in the Magnitude Range 11.5 - 16.0 around the Celestial Equator. Astronomical Journal, 104, 340-371. http://dx.doi.org/10.1086/116242

[12] Selim, I.M., Haroon, A.A., Ismail, H.A., Ahmed, N.M., Essam, A. and Ali, G.B. (2014) RAJ, $24,159$. 
[13] Bonatto, C., Bica, E. and Girardi, L. (2004) Theoretical Isochrones Compared to 2MASS Observations: Open Clusters at Nearly Solar Metallicity. Astronomy \& Astrophysics, 415, 571-576. http://dx.doi.org/10.1051/0004-6361:20034638

[14] Prisinzano, L., Micela, G., Sciortino, S. and Favata, F. (2003) Luminosity and Mass Function of the Galactic Open Cluster NGC 2422. Astronomy \& Astrophysics, 404, 927-937. http://dx.doi.org/10.1051/0004-6361:20030524

[15] Haroon, A., Ismail, H.A. and Alnagahy, F.Y. (2014) Two MASS Photometry of Open Star Clusters: King 13 and Berkeley 53. Astrophysics and Space Science, 352, 665-671. http://dx.doi.org/10.1007/s10509-014-1990-z

[16] King, I. (1966) The Structure of Star Clusters. IV. Photoelectric Surface Photometry in Nine Globular Clusters. Astronomical Journal, 71, 276. http://dx.doi.org/10.1086/109918

[17] Marigo, P., Girardi, L., Bressan, A., Groenewegen, M.A.T., Silva, L. and Granato, G.L. (2008) Evolution of Asymptotic Giant Branch Stars. II. Optical to Far-Infrared Isochrones with Improved TP-AGB Models. Astronomy \& Astrophysics, 482, 883-905. http://dx.doi.org/10.1051/0004-6361:20078467

[18] Spitzer Jr., L. and Hart, M.H. (1971) Random Gravitational Encounters and the Evolution of Spherical Systems. I. Method. Astrophysical Journal, 164, 399. http://dx.doi.org/10.1086/150855 